\title{
Sensibility Design Elements of Public Facilities for Improve Urban Image in Jeju: Focusing on Public Art Museums
}

\author{
SeoYoung $\mathrm{Kim}^{1}$ )
}

\begin{abstract}
Urban images can change the image of the city according to the way of expressing the environmental characteristics and cultural elements of the area. Art museums reflecting urban identity and cultural activities have an important influence on improving urban image. In this paper, improving the urban image by embodying the sensibility element of the public facilities of the museum which gives pleasure through cultural value. The categorization of sensibility design elements of public facilities was based on the elements of Donald Norman 's emotional design and Bernd Schmitt' s experience. As a research method, analyzed the present state of emotional design elements of public facilities in seven public art museums in Jeju. As a result of research, most of the public facilities in seven art museums have a stable emotion factor in the form of formalization. The emotional factors causing irregular fun and interest were not enough. Development of the olfactory and auditory sensibility elements is insufficient and development of the emotional element combined with IT technology is urgent.
\end{abstract}

Keywords : Sensibility Design Element, Public Facilities, The Role of the Art Museum, Cultural Value

\section{Introduction}

\subsection{Background and Purpose}

Art museums reflecting urban identity and cultural activities have an important influence on improving urban image. Art museums are expanding into the role of pursuing both cultural desires and pleasures as spaces that can satisfy various cultural needs. It is also a sociocultural basis that shows the quality of the region beyond the activation of the local economy. The higher the quality of life, the more varied the role of cultural facilities and the desire for emotional communication. The art museum needs a role to enrich its life through the creation of various cultures and emotional experiences as a space for exhibition and rest. There is a need for research to improve the urban image that embodies elements of pleasant emotional reflection reflecting the identity of public facilities in art museums. Sensibility design is a necessary factor for raising the quality of life of the members

Received(January 23, 2018), Review Result(1st: February 14, 2018, 2nd: February 28, 2018), Accepted(March 2, 2018)

1) (Professor) Dept. of Cultural Industry Mgmt., Honam University, Gwangju, Korea email: sykim@honam.ac.kr 
of the society and improving the image of the city to create economic value. The purpose of this study is to investigate and analyze the present state of the sensibility design elements of the public facilities installed in the art museum, and to utilize them as the basic data for creating the environment and culture for improving the urban image.

\subsection{Scope and Method}

This study extended the precedent study of 'Emotional Design Elements of Public Facilities: Focusing on public museums in Jeju' by SeoYoung Kim[1]. The study area is Jeju area, which has the most cultural facilities in Korea and has no research on public facilities. Among the top three cultural facilities (public libraries, art museums, and museums), the emotional design elements of public facilities were analyzed focusing on art museums, which are cultural facility spaces, which are expected to have high expectations for cultural creation and emotional experience . Scoping seven public art museums suitable for function and efficiency as a public space. The research method is to grasp the classification system of the public facilities and to summarize the theoretical concepts according to the classification system of the public facilities of the art museum. The emotional experience of sensory stimulation in public facilities was established based on the elements of design and psychologist Donald A. Norman's emotional design and Bernd Schmitt's experience. The tendency of sensibility design was derived by analyzing the state of sensibility design elements of public facilities in art museums.

\section{Theoretical Background}

\subsection{Urban Image, Public Facilities of Art Museum}

Urban images can change the image of the city according to the way of expressing the environmental characteristics and cultural elements of the area. Formation of urban identity is an important part of enhancing urban image and constituting competitiveness of city. Art museums reflecting urban identity and cultural activities have an important influence on improving urban image. Facilities in public art museums are facilities that are installed for all citizens based on functionality and safety. Public facilities in museums play an intermediary role in connecting people, space, and the environment. Today, the museum has expanded from the role of the exhibition space to the center of the cultural space that accommodates social and cultural demands[2]. Public facilities in Jeju are classified into lighting system facilities, resting facilities, sanitary facilities, traffic facilities, safety 
facilities, information facilities, guidance facilities, and other facilities[3]. Based on the criteria of public facilities in Jeju, the public facilities of art museums were classified into illumination facilities, relaxation facilities, sanitary facilities, information facilities, and other facilities[3].

[Table 1] Classification of Art Museum Public Facilities

\begin{tabular}{|c|l|l|}
\hline Space division & \multicolumn{1}{|c|}{ Main Category } & \multicolumn{1}{|c|}{ Detailed classification } \\
\hline \multirow{4}{*}{$\begin{array}{c}\text { internal space of } \\
\text { the art museum }\end{array}$} & relaxation facilities & bench \\
\cline { 2 - 3 } & sanitary facilities & trash can, water purifier, umbrella stand \\
\cline { 2 - 3 } & information facilities & notice board \\
\cline { 2 - 3 } & other facilities & locker \\
\hline \multirow{4}{*}{$\begin{array}{c}\text { external space } \\
\text { of art museum }\end{array}$} & illumination facilities & street lamp \\
\cline { 2 - 3 } & relaxation facilities & bench, pagoda \\
\cline { 2 - 3 } & sanitary facilities & trash can, water purifier \\
\cline { 2 - 3 } & information facilities & notice board, gate, sign \\
\hline
\end{tabular}

Public facilities are classified into inside space and outside space depending on the location of installation space. The inside space includes the entrance of the building, the exhibition space, the supporting facilities, and the outside space is the entrance sign, the rest room, the outdoor performance hall, and the garden[4-10].

\subsection{Sensibility design elements of public facilities}

Sensibility is the psychological sensibility of human being, which implies perception by the sensory functions of the five senses[11]. Sensibility design is a design that allows people to experience new emotions by stimulating their senses more sensitively, making them feel and revitalize their emotional memory. The role of sensibility design that conveys specific images by shaping the invisible sensibility or taste through visible colors, forms, and materials is becoming important[12]. The design factor of the public facilities is to induce the emotional response by experiencing the design elements that can stimulate the sensibility of the sensory function. Psychologist Bernd Schmitt classifies the elements of experience into five senses: sense, emotional feel, thinking think, act, relationship relate [13-14]. Sensory sense is a factor that focuses on enjoyment of the five senses of sight, hearing, touch, taste and smell[13-14]. It is a concept that can enhance the image of the brand through experience. Donald A. Norman's 'Emotional Design' book has classified emotional design into three dimensions. It is the human sense, the emotional visceral aspect, the behavioral aspect of usability centered design, and the reflective aspect of high dimensional thinking[15]. The instinctive design is an emotional response to the user's visual, taste, auditory, olfactory, and tactile sensations on the external 
appearance, which is the experience that the first feeling is formed. Sensibility design experiences sensibility through stimulation of the five senses. The sensibility design element of the public facilities is the role of inducing emotions formed by the stimulation of the five senses. However, due to the nature of public facilities, there are limitations in satisfying the five senses. Sensibility design elements of public facilities are classified into visual, auditory, tactile, and olfactory stimuli by sensory stimulation.

\section{Case study}

\subsection{Scope and Method}

There are 7 public art museums and 13 private art museums registered in Jeju. The public art museums include the Jeju Museum of Art, Jeju Museum of Contemporary Art, Leejungseob Art Museum, Gidang Art Museum[1], Chusa Museum, Soam Museum, and Kimtschangyeul Museum. Art museums are divided into interior and exterior spaces. The inside space is exhibition, and you can experience stable emotion and the outside space can experience free and comfortable feeling. The research scope of this study is divided into the public space facilities of the museums of 7 public museums[16] in Jeju area as the facilities inside the buildings and the facilities of the external spaces.

[Table 2] Case Study Space Classification

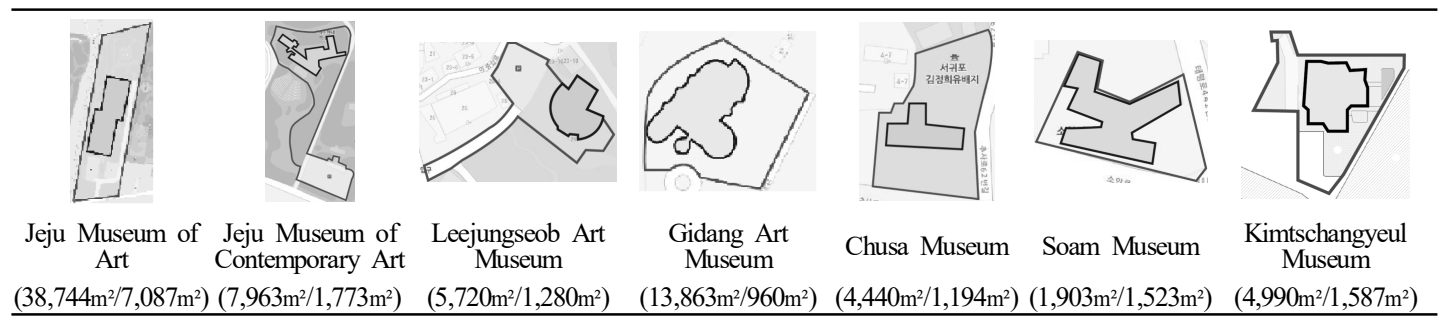

The scope of the public facilities of the museum is composed of 6 facilities in the internal space of the building and 8 facilities in the external space as public facilities with close interaction. The sensibility design elements of the public facilities in the museum were classified into visual elements, auditory elements, tactile elements and olfactory elements[1][13-15] through the preceding research.

Field surveys were conducted four times in June and September 2016 and January and July 2017, and then photographed directly with the camera, and interviewed the director of the museum and a curator. Other data were provided by telephone interviews with Jeju City Office and requesting data. 
[Table 3] Sensibility Design Element Classification of Public Facilities

\begin{tabular}{|c|c|c|c|c|c|c|c|c|}
\hline $\begin{array}{l}\text { sensibility } \\
\text { design }\end{array}$ & Desig & $\begin{array}{l}\text { elements of public } \\
\text { facilities }\end{array}$ & $\begin{array}{l}\text { sensibility } \\
\text { design }\end{array}$ & Design & $\begin{array}{l}\text { ments of public } \\
\text { cilities }\end{array}$ & $\begin{array}{l}\text { sensibility } \\
\text { design }\end{array}$ & Desig & $\begin{array}{l}\text { elements of public } \\
\text { facilities }\end{array}$ \\
\hline \multirow{6}{*}{$\begin{array}{l}\text { Visual } \\
\text { element }\end{array}$} & \multirow[b]{2}{*}{ Color } & \multirow[b]{2}{*}{ Color combination } & \multirow{6}{*}{$\begin{array}{l}\text { Tactile } \\
\text { element }\end{array}$} & \multirow{6}{*}{ Texture } & Metal & \multirow{3}{*}{$\begin{array}{l}\text { Auditory } \\
\text { element }\end{array}$} & Voice & Language voice \\
\hline & & & & & Plastic & & Music & Background music \\
\hline & \multirow{4}{*}{ Shape } & Natural color & & & Glass & & $\begin{array}{l}\text { Natural } \\
\text { sound }\end{array}$ & $\begin{array}{c}\text { A primordial sound } \\
\text { of nature }\end{array}$ \\
\hline & & Formalization & & & Fabric & \multirow{3}{*}{$\begin{array}{l}\text { Olfactory } \\
\text { element }\end{array}$} & \multirow{3}{*}{ Scent } & Natural incense \\
\hline & & \multirow{2}{*}{ Informalization } & & & $\begin{array}{c}\text { Environment } \\
\text { materials }\end{array}$ & & & Incense of material \\
\hline & & & & & Cement & & & Artificial fragrance \\
\hline
\end{tabular}

\subsection{Field survey}

There are Jeju Museum of Art, Jeju Museum of Contemporary Art, Kimtschangyeul Museum in Jeju City, Leejungseob Art Museum, Gidang Art Museum, Chusa Museum, Soam Museum in Seogwipo City. Leejungseob Art Museum and Soam Museum are located in Seogwipo downtown area and other art museums are located in open spaces in nature. The museum, located in the city center, is convenient to visit because of its convenient accessibility but lacks external space and has low harmony with nature. In the current field survey, extended the previous research on 'Emotional Design Elements of Public Facilities: Focusing on public museums in Jeju' by SeoYoung Kim (2017). [Table 4] shows the field survey of the emotional design elements of public facilities in seven public museums.

[Table 4] Investigation Status

\begin{tabular}{|c|c|c|c|c|c|c|c|c|c|c|c|}
\hline \multicolumn{6}{|c|}{ Jeju Museum of Art } & \multicolumn{6}{|c|}{ Jeju Museum of Contemporary Art } \\
\hline \multicolumn{2}{|c|}{ facility } & \multirow{2}{*}{$\begin{array}{c}\begin{array}{c}\text { Visual } \\
\text { element }\end{array} \\
2 \text { colors/ } \\
\text { Formal }\end{array}$} & \multirow{2}{*}{$\begin{array}{c}\begin{array}{c}\text { Auditory } \\
\text { element }\end{array} \\
\text { fricative }\end{array}$} & \multirow{2}{*}{\begin{tabular}{|c|c|c|}
$\begin{array}{c}\text { Tactile } \\
\text { element }\end{array}$ \\
$\begin{array}{c}\text { metal+ } \\
\text { Leatherette }\end{array}$ \\
\end{tabular}} & \multirow{2}{*}{$\begin{array}{c}\begin{array}{c}\text { Olfactory } \\
\text { element }\end{array} \\
\begin{array}{c}\text { Incense of } \\
\text { material }\end{array}\end{array}$} & \multicolumn{2}{|c|}{ facility } & \multirow{2}{*}{$\begin{array}{c}\begin{array}{c}\text { Visual } \\
\text { element }\end{array} \\
1-3 \text { colors/ } \\
\text { Formal }\end{array}$} & \multirow{2}{*}{$\begin{array}{c}\begin{array}{c}\text { Auditory } \\
\text { element }\end{array} \\
-\end{array}$} & \multirow{2}{*}{\begin{tabular}{|c|}
$\begin{array}{c}\text { Tactile } \\
\text { element }\end{array}$ \\
$\begin{array}{c}\text { fabric }+ \text { meta, } \\
\text { wood, stone }\end{array}$ \\
\end{tabular}} & \multirow{2}{*}{$\begin{array}{c}\begin{array}{c}\text { Olfactory } \\
\text { element }\end{array} \\
-\end{array}$} \\
\hline \multirow{6}{*}{$\begin{array}{c}\text { internal } \\
\text { space }\end{array}$} & bench & & & & & \multirow{6}{*}{$\begin{array}{c}\text { internal } \\
\text { space }\end{array}$} & bench & & & & \\
\hline & trash can & $\begin{array}{c}2 \text { colors/ } \\
\text { Formal }\end{array}$ & - & plastic & - & & trash can & $\begin{array}{c}1 \text { colors/ } \\
\text { Formal }\end{array}$ & fricative & metal & - \\
\hline & $\begin{array}{c}\text { water } \\
\text { purifier }\end{array}$ & $\begin{array}{l}2 \text { colors/ } \\
\text { Formal }\end{array}$ & fricative & mix plastic & $\begin{array}{l}\text { Natural } \\
\text { incense }\end{array}$ & & $\begin{array}{c}\text { water } \\
\text { purifier }\end{array}$ & $\begin{array}{l}1 \text { colors/ } \\
\text { Formal }\end{array}$ & fricative & plastic & $\begin{array}{l}\text { Natural } \\
\text { incense }\end{array}$ \\
\hline & $\begin{array}{c}\text { umbrella } \\
\text { stand }\end{array}$ & $\begin{array}{l}\text { 1-2 colors/ } \\
\text { Formal }\end{array}$ & - & $\begin{array}{c}\text { metal+plastic, } \\
\text { metal+wood, } \\
\text { metal }\end{array}$ & - & & $\begin{array}{c}\text { umbrella } \\
\text { stand }\end{array}$ & $\begin{array}{l}2 \text { colors/ } \\
\text { Formal }\end{array}$ & - & $\underset{\text { metal }}{\text { metal+plastic }}$ & - \\
\hline & board & $\begin{array}{c}\text { 1-3 colors/ } \\
\text { Formal }\end{array}$ & - & $\begin{array}{c}\text { wood, plastic, } \\
\text { cement }\end{array}$ & - & & board & $\begin{array}{l}1 \text { colors/ } \\
\text { Formal }\end{array}$ & - & wood & - \\
\hline & locker & $\begin{array}{l}2 \text { colors/ } \\
\text { Formal }\end{array}$ & - & wood + metal & - & & locker & $\begin{array}{l}1 \text { colors/ } \\
\text { Formal }\end{array}$ & - & wood & - \\
\hline \multirow{4}{*}{$\begin{array}{c}\text { external } \\
\text { space }\end{array}$} & $\begin{array}{l}\text { street } \\
\text { lamp }\end{array}$ & $\begin{array}{l}\text { 1-2 colors/ } \\
\text { Formal }\end{array}$ & BG music & metal+glass & - & \multirow{4}{*}{$\begin{array}{c}\text { external } \\
\text { space }\end{array}$} & $\begin{array}{l}\text { street } \\
\text { lamp }\end{array}$ & $\begin{array}{l}1 \text { colors/ } \\
\text { Formal }\end{array}$ & $\begin{array}{l}\text { BG music } \\
\text { sound of } \\
\text { nature }\end{array}$ & metal, glass & - \\
\hline & bench & $\begin{array}{l}1 \text { colors/ } \\
\text { Forma- } \\
\text { Informal }\end{array}$ & - & stone & $\begin{array}{c}\text { Incense of } \\
\text { material }\end{array}$ & & bench & $\begin{array}{l}\text { 1-2 colors/ } \\
\text { Forma-1 } \\
\text { Informal }\end{array}$ & - & $\begin{array}{l}\text { woo+metal, } \\
\text { stone, wood }\end{array}$ & $\begin{array}{c}\text { Incense of } \\
\text { material }\end{array}$ \\
\hline & pagoda & $\begin{array}{c}\text { 1-2 colors/ } \\
\text { Formal }\end{array}$ & - & wood+stone & $\begin{array}{c}\text { Incense of } \\
\text { material }\end{array}$ & & pagoda & $\begin{array}{c}1 \text { colors/ } \\
\text { Formal }\end{array}$ & $\begin{array}{l}\text { Background } \\
\text { music }\end{array}$ & wood & $\begin{array}{c}\text { Incense of } \\
\text { material }\end{array}$ \\
\hline & trash can & $\begin{array}{l}\text { 1-2 colors/ } \\
\text { Formal }\end{array}$ & fricative & metal & - & & trash can & $\begin{array}{l}\text { 1-2 colors/ } \\
\text { Formal }\end{array}$ & fricative & metal, wood & - \\
\hline
\end{tabular}




\begin{tabular}{|c|c|c|c|c|c|c|c|c|c|c|c|}
\hline & $\begin{array}{c}\text { water } \\
\text { purifier }\end{array}$ & $\begin{array}{l}1 \text { colors/ } \\
\text { Formal }\end{array}$ & - & stone + metal & - & & $\begin{array}{c}\text { water } \\
\text { purifier }\end{array}$ & - & - & - & - \\
\hline & board & $\begin{array}{l}2 \text { colors/ } \\
\text { Formal }\end{array}$ & - & \begin{tabular}{|c|} 
metal+glass + \\
stone +
\end{tabular} & - & & board & $\begin{array}{l}2 \text { colors/ } \\
\text { Formal }\end{array}$ & - & $\begin{array}{c}\text { metal+glass } \\
\text { +wood }\end{array}$ & - \\
\hline & gate & $\begin{array}{c}2 \text { colors/ } \\
\text { Formal }\end{array}$ & - & stone+wood & - & & gate & $\begin{array}{l}2 \text { colors/ } \\
\text { Formal }\end{array}$ & - & stone+wood & - \\
\hline & sign & $\begin{array}{l}5 \text { colors/ } \\
\text { Formal }\end{array}$ & - & stone + metal & - & & sign & $\begin{array}{l}2 \text { colors/ } \\
\text { Formal }\end{array}$ & - & stone & - \\
\hline \multicolumn{6}{|c|}{ Leejungseob Art Museum } & \multicolumn{6}{|c|}{ Gidang Art Museum } \\
\hline \multicolumn{2}{|c|}{ facility } & $\begin{array}{l}\text { Visual } \\
\text { element }\end{array}$ & $\begin{array}{c}\text { Auditory } \\
\text { element }\end{array}$ & $\begin{array}{c}\text { Tactile } \\
\text { element }\end{array}$ & $\begin{array}{l}\text { Olfactory } \\
\text { element }\end{array}$ & \multicolumn{2}{|c|}{ facility } & $\begin{array}{l}\text { Visual } \\
\text { element }\end{array}$ & $\begin{array}{c}\text { Auditory } \\
\text { element }\end{array}$ & $\begin{array}{c}\text { Tactile } \\
\text { element }\end{array}$ & $\begin{array}{l}\text { Olfactory } \\
\text { element }\end{array}$ \\
\hline \multirow{6}{*}{$\begin{array}{c}\text { internal } \\
\text { space }\end{array}$} & bench & - & - & - & - & \multirow{6}{*}{$\begin{array}{c}\text { internal } \\
\text { space }\end{array}$} & bench & $\begin{array}{l}1 \text { colors/ } \\
\text { Formal }\end{array}$ & - & wood & $\begin{array}{c}\text { Incense of } \\
\text { material }\end{array}$ \\
\hline & trash can & $\begin{array}{l}1 \text { colors/ } \\
\text { Formal }\end{array}$ & fricative & metal & - & & trash can & $\begin{array}{l}4 \text { colors/ } \\
\text { Formal }\end{array}$ & fricative & metal & - \\
\hline & $\begin{array}{c}\text { water } \\
\text { purifier }\end{array}$ & $\begin{array}{l}1 \text { colors/ } \\
\text { Formal }\end{array}$ & fricative & mix plastic & $\begin{array}{l}\text { Natural } \\
\text { incense }\end{array}$ & & $\begin{array}{c}\text { water } \\
\text { purifier }\end{array}$ & $\begin{array}{l}1 \text { colors/ } \\
\text { Formal }\end{array}$ & - & metal & - \\
\hline & $\begin{array}{c}\text { umbrella } \\
\text { stand }\end{array}$ & $\begin{array}{c}2 \text { colors/ } \\
\text { Formal }\end{array}$ & - & metal + plastic & - & & $\begin{array}{c}\text { umbrella } \\
\text { stand }\end{array}$ & $\begin{array}{l}1 \text { colors/ } \\
\text { Formal }\end{array}$ & - & metal & - \\
\hline & board & - & - & - & - & & board & - & - & - & - \\
\hline & locker & $\begin{array}{l}1 \text { colors/ } \\
\text { Formal }\end{array}$ & - & wood & - & & locker & $\begin{array}{l}1 \text { colors/ } \\
\text { Formal }\end{array}$ & - & wood & - \\
\hline \multirow{8}{*}{$\begin{array}{c}\text { external } \\
\text { space }\end{array}$} & $\begin{array}{l}\text { street } \\
\text { lamp }\end{array}$ & $\begin{array}{l}1 \text { colors/ } \\
\text { Formal }\end{array}$ & $\begin{array}{l}\text { Background } \\
\text { music }\end{array}$ & metal+glass & - & \multirow{8}{*}{$\begin{array}{c}\text { external } \\
\text { space }\end{array}$} & $\begin{array}{l}\text { street } \\
\text { lamp }\end{array}$ & $\begin{array}{c}2 \text { colors/ } \\
\text { Formal }\end{array}$ & - & metal, glass & - \\
\hline & bench & $\begin{array}{l}1 \text { colors/ } \\
\text { Formal }\end{array}$ & fricative & wood & $\begin{array}{c}\text { Incense of } \\
\text { material }\end{array}$ & & bench & $\begin{array}{l}1 \text { colors/ } \\
\text { Formal }\end{array}$ & - & cement & - \\
\hline & pagoda & $\begin{array}{l}1 \text { colors/ } \\
\text { Formal }\end{array}$ & - & $\begin{array}{c}\text { wood }+ \\
\text { meta }\end{array}$ & $\begin{array}{c}\text { Incense of } \\
\text { material }\end{array}$ & & pagoda & $\begin{array}{l}1 \text { colors/ } \\
\text { Formal }\end{array}$ & - & cement & - \\
\hline & trash can & - & - & - & - & & trash can & - & - & - & - \\
\hline & \begin{tabular}{|c|}
$\begin{array}{c}\text { water } \\
\text { purifier }\end{array}$ \\
\end{tabular} & - & - & - & - & & \begin{tabular}{|c|}
$\begin{array}{c}\text { water } \\
\text { purifier }\end{array}$ \\
\end{tabular} & - & - & - & - \\
\hline & board & - & - & - & - & & board & - & - & - & - \\
\hline & gate & $\begin{array}{l}2 \text { colors/ } \\
\text { Formal }\end{array}$ & - & stone+wood & - & & gate & $\begin{array}{l}2 \text { colors/ } \\
\text { Formal }\end{array}$ & - & stone+wood & - \\
\hline & sign & $\begin{array}{l}2 \text { colors/ } \\
\text { Formal }\end{array}$ & - & stone + metal & - & & sign & $\begin{array}{l}1 \text { colors/ } \\
\text { Formal }\end{array}$ & - & metal & - \\
\hline \multicolumn{6}{|c|}{ Chusa Museum } & \multicolumn{6}{|c|}{ Soam Museum } \\
\hline \multicolumn{2}{|c|}{ facility } & $\begin{array}{l}\text { Visual } \\
\text { element }\end{array}$ & $\begin{array}{c}\text { Auditory } \\
\text { element }\end{array}$ & $\begin{array}{c}\text { Tactile } \\
\text { element }\end{array}$ & $\begin{array}{c}\text { Olfactory } \\
\text { element }\end{array}$ & \multicolumn{2}{|c|}{ facility } & $\begin{array}{l}\text { Visual } \\
\text { element }\end{array}$ & $\begin{array}{c}\text { Auditory } \\
\text { element }\end{array}$ & $\begin{array}{l}\text { Tactile } \\
\text { element }\end{array}$ & $\begin{array}{c}\text { Olfactory } \\
\text { element }\end{array}$ \\
\hline \multirow{6}{*}{$\begin{array}{c}\text { internal } \\
\text { space }\end{array}$} & bench & $\begin{array}{l}2 \text { colors/ } \\
\text { Formal }\end{array}$ & fricative & $\begin{array}{c}\text { metal+ } \\
\text { Leatherette }\end{array}$ & $\begin{array}{c}\text { Incense of } \\
\text { material }\end{array}$ & \multirow{6}{*}{$\begin{array}{c}\text { internal } \\
\text { space }\end{array}$} & bench & $\begin{array}{l}2 \text { colors/ } \\
\text { Formal }\end{array}$ & fricative & meta+fabicl, & $\begin{array}{c}\text { Incense of } \\
\text { material }\end{array}$ \\
\hline & trash can & $\begin{array}{l}1 \text { colors/ } \\
\text { Formal }\end{array}$ & fricative & metal & & & trash can & $\begin{array}{l}2 \text { colors/ } \\
\text { Formal }\end{array}$ & fricative & plastic & - \\
\hline & \begin{tabular}{|c|} 
water \\
purifier
\end{tabular} & $\begin{array}{l}1 \text { colors/ } \\
\text { Formal }\end{array}$ & fricative & mix plastic & $\begin{array}{l}\text { Natural } \\
\text { incense }\end{array}$ & & $\begin{array}{c}\text { water } \\
\text { purifier }\end{array}$ & $\begin{array}{l}1 \text { colors/ } \\
\text { Formal }\end{array}$ & fricative & & - \\
\hline & $\begin{array}{c}\text { umbrella } \\
\text { stand }\end{array}$ & $\begin{array}{l}1 \text { colors/ } \\
\text { Formal }\end{array}$ & fricative & metal & & & $\begin{array}{c}\text { umbrella } \\
\text { stand }\end{array}$ & $\begin{array}{l}1 \text { colors/ } \\
\text { inFormal }\end{array}$ & fricative & stone & - \\
\hline & board & - & - & - & - & & board & $\begin{array}{c}\text { 1-3 colors/ } \\
\text { Formal }\end{array}$ & - & $\begin{array}{l}\text { metal+glass }+ \\
\text { stone }\end{array}$ & - \\
\hline & locker & - & - & - & - & & locker & - & - & - & - \\
\hline \multirow{8}{*}{$\begin{array}{c}\text { external } \\
\text { space }\end{array} \mid$} & $\begin{array}{l}\text { street } \\
\text { lamp }\end{array}$ & $\begin{array}{l}2 \text { colors/ } \\
\text { Formal }\end{array}$ & - & metal+glass & - & \multirow{8}{*}{$\begin{array}{c}\text { external } \\
\text { space }\end{array}$} & $\begin{array}{l}\text { street } \\
\text { lamp }\end{array}$ & $\begin{array}{l}3 \text { colors/ } \\
\text { Formal }\end{array}$ & - & \begin{tabular}{|c|} 
wood + metal + \\
glass, \\
metal + glass \\
\end{tabular} & - \\
\hline & bench & $\begin{array}{l}2 \text { colors/ } \\
\text { Formal }\end{array}$ & fricative & wood + metal & $\begin{array}{c}\text { Incense of } \\
\text { material }\end{array}$ & & bench & $\begin{array}{l}2 \text { colors/ } \\
\text { Formal }\end{array}$ & fricative & $\begin{array}{c}\text { stone, wood } \\
\text { wood +metal }\end{array}$ & $\begin{array}{c}\text { Incense of } \\
\text { material }\end{array}$ \\
\hline & pagoda & $\begin{array}{l}1 \text { colors/ } \\
\text { Formal }\end{array}$ & - & $\begin{array}{c}\text { wood }+ \\
\text { meta }\end{array}$ & $\begin{array}{c}\text { Incense of } \\
\text { material }\end{array}$ & & pagoda & - & - & - & - \\
\hline & trash can & - & - & - & - & & trash can & $\begin{array}{l}1 \text { colors/ } \\
\text { inFormal }\end{array}$ & fricative & stone & - \\
\hline & \begin{tabular}{|c|} 
water \\
purifier
\end{tabular} & $\begin{array}{l}1 \text { colors/ } \\
\text { inFormal }\end{array}$ & - & stone + metal & & & \begin{tabular}{|c|} 
water \\
purifier
\end{tabular} & - & fricative & - & - \\
\hline & board & - & - & - & - & & board & - & - & - & - \\
\hline & gate & $\begin{array}{l}2 \text { colors/ } \\
\text { Formal }\end{array}$ & - & stone+wood & - & & gate & - & - & - & - \\
\hline & sign & $\begin{array}{l}2 \text { colors/ } \\
\text { Formal }\end{array}$ & - & metal & - & & sign & $\begin{array}{l}1 \text { colors/ } \\
\text { Formal }\end{array}$ & - & metal & - \\
\hline \multicolumn{12}{|c|}{ Kimtschangyeul Museum } \\
\hline \multicolumn{2}{|c|}{ facility } & Visual & Auditory & Tactile & Olfactory & & cility & Visual & Auditory & Tactile & Olfactory \\
\hline
\end{tabular}


Asia-pacific Journal of Convergent Research Interchange

Vol.4, No.1, March 31 (2018), pp. 69-79

http://dx.doi.org/10.14257/apjcri.2018.03.08

\begin{tabular}{|c|c|c|c|c|c|c|c|c|c|c|c|}
\hline & & element & element & element & element & & & element & element & element & element \\
\hline \multirow{8}{*}{$\begin{array}{c}\text { internal } \\
\text { space }\end{array}$} & bench & $\begin{array}{c}2 \text { colors/ } \\
\text { Formal }\end{array}$ & fricative & metal & - & \multirow{8}{*}{$\begin{array}{c}\text { external } \\
\text { space }\end{array}$} & $\begin{array}{l}\text { street } \\
\text { lamp }\end{array}$ & $\begin{array}{c}1 \text { colors/ } \\
\text { Formal }\end{array}$ & - & metal & - \\
\hline & trash can & $\begin{array}{l}\text { 1_colors/ } \\
\text { Formal }\end{array}$ & fricative & plastic & - & & bench & $\begin{array}{c}2 \text { colors/ } \\
\text { Formal }\end{array}$ & fricative & $\begin{array}{c}\text { wood+metal }+ \\
\text { stone }\end{array}$ & $\begin{array}{c}\text { Incense of } \\
\text { material }\end{array}$ \\
\hline & $\begin{array}{c}\text { water } \\
\text { purifier }\end{array}$ & $\begin{array}{c}1 \text { colors/ } \\
\text { Formal }\end{array}$ & fricative & mix plastic & $\begin{array}{l}\text { Natural } \\
\text { incense }\end{array}$ & & pagoda & - & - & - & - \\
\hline & $\begin{array}{c}\text { umbrella } \\
\text { stand }\end{array}$ & $\begin{array}{c}\text { 1 colors/ } \\
\text { Formal }\end{array}$ & fricative & metal & & & trash can & - & - & - & - \\
\hline & \multirow{2}{*}{ board } & \multirow{2}{*}{ - } & \multirow{2}{*}{ - } & \multirow{2}{*}{-} & \multirow{2}{*}{ - } & & $\begin{array}{c}\text { water } \\
\text { purifier }\end{array}$ & - & - & - & - \\
\hline & & & & & & & board & - & - & - & - \\
\hline & \multirow[b]{2}{*}{ locker } & \multirow{2}{*}{$\begin{array}{l}\text { 1 colors/ } \\
\text { Formal }\end{array}$} & \multirow[b]{2}{*}{ - } & \multirow[b]{2}{*}{ metal } & \multirow[b]{2}{*}{ - } & & gate & - & - & - & - \\
\hline & & & & & & & sign & $\begin{array}{c}3 \text { colors/ } \\
\text { Formal }\end{array}$ & - & metal & - \\
\hline
\end{tabular}

Jeju Museum of Art is the only museum with 14 public facilities and Kimtschangyeul Museum has the smallest 10 facilities. Kimtschangyeul Museum opened in September, 2016, and the outdoor space is still being maintained.

\section{Analysis}

Based on the field survey, two analysis cases of representative art museums were analyzed as an analysis framework for the sensibility design elements. The representative museum cases were selected based on the area of art museum, the number of visitors, and the number of installed facilities.

1) Jeju Museum of Art

Jeju Museum of Art was opened in 2009[4] and is the representative museum of Jeju area. It is located in Jeju City and has good accessibility and the largest area of art museum. In particular, outdoor gardens and outdoor stage facilities are well equipped.

[Table 5] Analysis of Public Facilities in Jeju Museum of Art

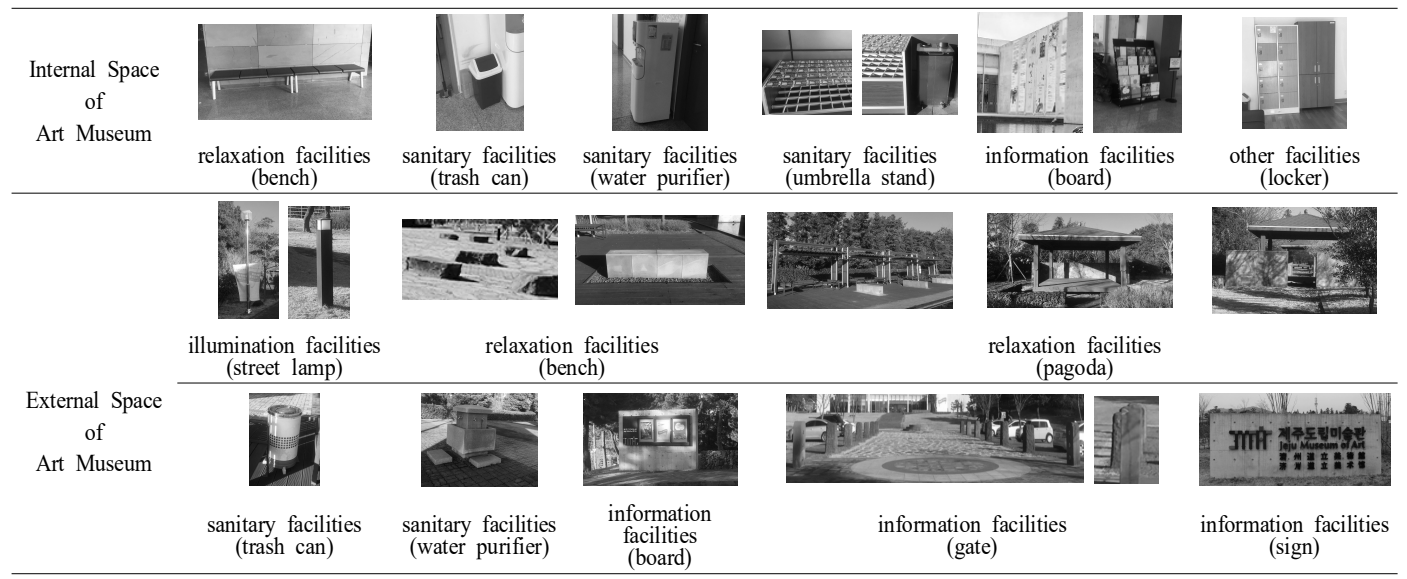




\section{(1) Visual element}

Facilities in the inner space of the museum are shaped by a simple and simple design. About eighty percent of the facilities are two or less colors and low saturation. Only the trash can facilities were blue and white in a combination of high saturation colors and were not in harmony with the interior space. Facilities in the external space of the museum coexisted with a simple design style and a unique non-standard style. In particular, the stone bench is a visual element that expresses the identity of the area as Baengnokdam shape.

(2) Tactile element

The materials of the public facilities were interesting by using various textures of metal, concrete, glass, cloth, plastic, wood and stone. Fifty percent of the facilities are represented by local identities using eco-friendly wood, stone, and basalt. The trash cans and water purifier in the inner space are made of plastic materials, and they are not in harmony with the surroundings and have no identity.

(3) Auditory element

The auditory elements are mainly expressed in outer space. Jeju Museum of Art is rich in the sounds of nature that can delight in the nature of Jeju and its atmosphere can be calmly relieved by its spacious exterior. The streetlight facility is equipped with speakers to produce quiet background music.

(4) Olfactory element

The olfactory element is the incense coming from the material of the facility and the natural smell coming from nature. No artificial fragrance is used at all.

2) Jeju Museum of Contemporary Art

Jeju Museum of Modern Art opened in 2007[5] and consists of main building and branch. Accessibility is poor, but the natural environment is well established.

(1) Visual element

Facilities in the interior space of the museum are shaped in a simple design, but bench is unconformable and the colors are blue, red, and natural, with no identity and no match with the surrounding environment. The facilities in the external space of the museum are a simple form of formal design, but various types are interesting.

(2) Tactile element

The materials of the public facilities were variously used in the texture of metal, concrete, glass, cloth, plastic, wood and stone. Relaxation facilities in the outer space are all made of environment-friendly materials such as wood and stone(basalt), and are harmonious with the natural environment. 
[Table 6] Analysis of Public Facilities in Jeju Museum of Contemporary Art

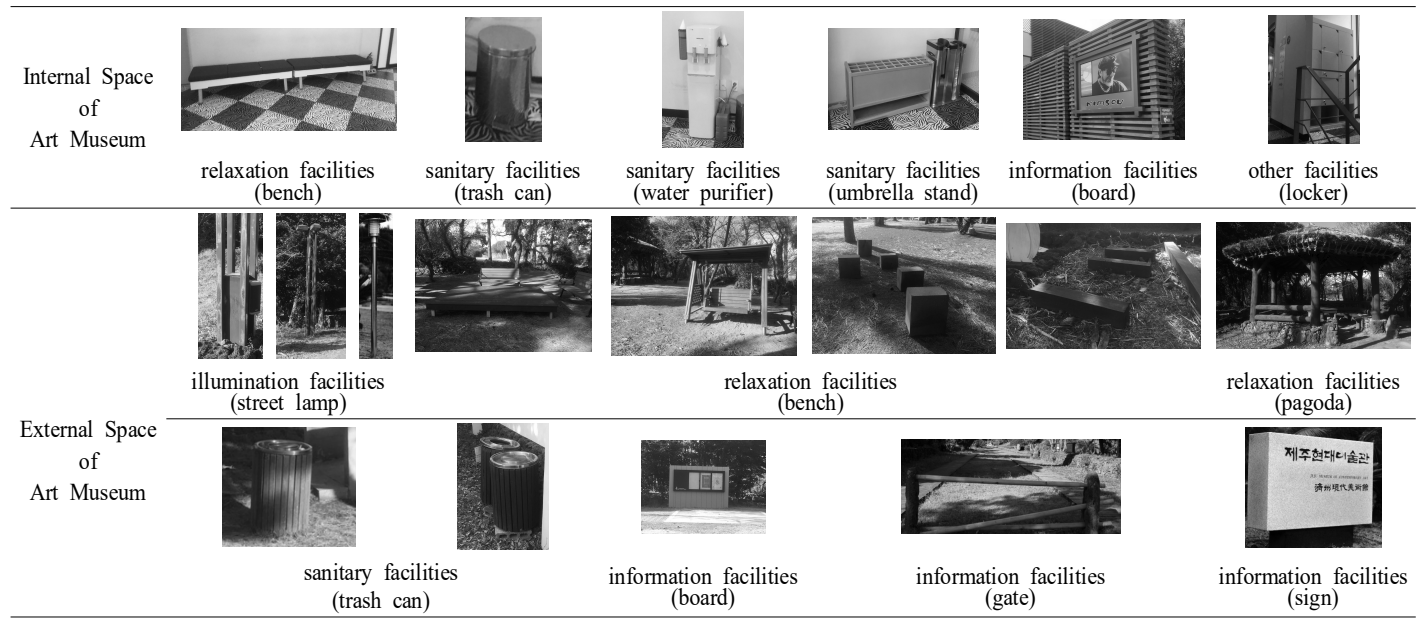

\section{(3) Auditory element}

The outside space is full of nature sounds that can enjoy nature of Jeju. Especially, I installed a new house on the tree and heard a lot of new sounds. The street lamps are equipped with loudspeakers, and speakers made of paper that express the basalt feel under the trees are harmonious with the surroundings and express the local identity.

(4) Olfactory element

The olfactory elements include wood fragrances from the materials of the facility and natural fragrances from the garden.

\section{Conclusion}

Urban images can change the image of the city according to the way of expressing the environmental characteristics and cultural elements of the area. Art museums reflecting urban identity and cultural activities have an important influence on improving urban image.

The purpose of this paper is to develop the environment and culture for urban image enhancement through the design elements of the sensitively design of public museums in Jeju area. First, the sanitary facilities in the inner space of all 7 museums are using the products of outside companies. Opening the older the museum, the more refractory materials are installed. There are no gates at Soam and Kimtschangyeul's art museums, but all other gates have a 'jeongnang'. Second, although the internal space facilities have a stable emotional element, the expression of the identity of the area is insufficient. The external space facilities are shyly reflected in the identity of the area and the 
element of emotional sensitivity. However, there are few stimulating factors that cause uniqueness and irregular interest and fun. Third, development of olfactory and auditory sensibility elements is urgent, and it is urgent to develop sensibility elements combined with IT technology. Fourth, the environment for improvement of urban image is created through the trend of sensitivity design for art museum facilities. It will be a basic study of the function and role improvement of the public facilities of the art museum which will be developed later.

\section{Acknowledgement}

This paper was supported by Research Grant from Honam University in 2016

\section{References}

[1] Kim, SeoYoung, Emotional Design Elements of Public Facilities -Focusing on public museums in Jeju, International Journal of ICT-aided Architecture and Civil Engineering, (2017), Vol.4, No.2, pp.45-50.

[2] Lee, Hyun Seo, Hur, Bum Pall, A Study on the Characteristics of Spatial Elements Exhibited in Art Museum Designed by SANAA, Korean Institute of Spatial Design, (2014), Vol.28, pp.160.

[3] City design of Jeju, Standard design of street facilities, Jeju Special Self-Governing Province, (2012)

[4] http://jmoa.jeju.go.kr, Oct 17 (2017)

[5] http://www.jejumuseum.go.kr, Oct 25 (2017)

[6] http://culture.seogwipo.go.kr/jslee, Oct 25 (2017)

[7] http://culture.seogwipo.go.kr/gidang, Oct 25 (2017)

[8] http://culture.seogwipo.go.kr/soam, Dec 27 (2017)

[9] http://www.jeju.go.kr/chusa, Dec. 27 (2017)

[10] http://kimtschang-yeul.jeju.go.kr/kimArt, Dec. 27 (2017)

[11] Kim, mijija, Emotional Engineering, Design Office, Korea, (1998), pp.4-5.

[12] Baek, Ji Seon, A Study on the Sensitivity Elements in Design of E-Brand Site : Focusing on Mobile Phone brand site, The Graduate School of Information Science, Ewha Womans University, (2008), pp.22-23.

[13] Schmitt, H. Bernd, Experiential Marketing: How to Get Customers to Sense, Feel, Think, Act, Free Press, USA, (1999). pp.16-149.

[14] Kim Eun-a, Urban Design Management, Korean Studies Information, (2009), pp.104-105. 
[15] Norman, A. Donald, Emotional Design: Why We Love [or hate] Everyday Things, Hakjisa, Korea, (2011), pp.87-127.

[16] http://map.daum.net, Feb 8 (2017) 